ANNALES

POLONICI MATHEMATICI

91.2-3 (2007)

\title{
The gradient lemma
}

\author{
by Urban Cegrell (Umeå and Sundsvall)
}

Abstract. We show that if a decreasing sequence of subharmonic functions converges to a function in $W_{\text {loc }}^{1,2}$ then the convergence is in $W_{\mathrm{loc}}^{1,2}$.

1. Introduction. This paper is based on a talk I gave in Kraków on April 30, 2003 and is in part motivated by Błocki's paper [1].

Proposition 1.1. Denote by $\mathrm{SH}^{-}$the negative subharmonic functions defined on some domain in $\mathbb{C}^{n}$, and by $W_{\text {loc }}^{1,2}$ the usual Sobolev class. Then $u \in \mathrm{SH}^{-} \cap W_{\text {loc }}^{1,2}$ if and only if $u \in \mathrm{SH}^{-} \cap L_{\text {loc }}^{1}(\Delta u)$.

Using Proposition 1.1, we prove the gradient lemma:

LEMMA 1.2. If $u_{j}$ is a decreasing sequence of functions in $\mathrm{SH}^{-}$with limit $u \in W_{\mathrm{loc}}^{1,2}$, then $u_{j} \in W_{\mathrm{loc}}^{1,2}$ and $u_{j} \rightarrow u$ in $W_{\mathrm{loc}}^{1,2}$ as $j \rightarrow \infty$.

In the last section, we use the gradient lemma in connection with the class $\mathcal{E}$.

2. Proof of Proposition 1.1. The problem is local, so we can assume that $u \in \mathrm{SH}^{-}(B)$ where $B$ is the unit ball in $\mathbb{C}^{n}, 0<r<s<1$. Define $\widetilde{u}=\sup \left\{\varphi \in \mathrm{SH}^{-}(B) ;\left.\varphi\right|_{r B} \leq\left. u\right|_{r B}\right\}$. Then $0 \geq \widetilde{u} \geq u, \widetilde{u} \in \mathrm{SH}^{-}(B), \widetilde{u}=u$ on $r B$ and $\widetilde{u}$ is harmonic on $B \backslash r B$ and $\widetilde{u}(x)=\int_{s B} g(x, y) \Delta \widetilde{u}(y)$ where $g$ is the Green function for $B$.

The smallest harmonic majorant of $u$ on $s B$ can be estimated from below on $r B$ by $c \int u d v$ where $c$ is a positive constant (depending on $s$ and $t$ ) and $d v$ is the Lebesgue measure on $B$. It follows that

$$
\int_{s B} g(x, y) \Delta u(y)+c \int_{B} u d v \leq u \quad \text { on } r B .
$$

2000 Mathematics Subject Classification: 32U05, 31B05.

Key words and phrases: subharmonic functions, plurisubharmonic functions, Sobolev norm. 
For $x \in B$, we have

$$
0 \geq \widetilde{u}(x) \geq \int_{s B} g(x, y) \Delta u(y)+c \frac{|x|^{2}-1}{r^{2}-1} \int_{B} u d v=: \bar{u}(x) .
$$

Since

$$
\int_{B}\left(1-|x|^{2}\right) \Delta \widetilde{u}=\int_{B}-\widetilde{u} d v
$$

we get

$$
\begin{aligned}
\int_{B}|\operatorname{grad} \widetilde{u}|^{2} d v & =\int_{B}-\widetilde{u} \Delta \widetilde{u} \leq \int_{B}-\bar{u} \Delta \widetilde{u} \\
& =\int_{B}\left\{\int_{s B}-g(x, y) \Delta u(y)\right\} \Delta \widetilde{u}+\frac{c}{r^{2}-1} \int_{B} u d v \int_{B}\left(1-|x|^{2}\right) \Delta \widetilde{u} \\
& \leq \int_{s B}-\widetilde{u} \Delta u+\frac{c}{1-r^{2}}\left(\int_{B} u d v\right)^{2} \leq \int_{s B}-u \Delta u+\frac{c}{1-r^{2}}\left(\int_{B} u d v\right)^{2}
\end{aligned}
$$

so if $\int_{s B}-u \Delta u<\infty$, then

$$
\int_{r B}|\operatorname{grad} u|^{2} \leq \int_{B}|\operatorname{grad} \widetilde{u}|^{2} \leq \int_{s B}-u \Delta u+\frac{c}{1-r^{2}}\left(\int_{B} u d v\right)^{2}
$$

and we have proved the first half of Proposition 1.1.

Assume now that $u \in \mathrm{SH}^{-} \cap W_{\text {loc }}^{1,2}$. We prove that then $\int_{r B}-u \Delta u<\infty$. Let $0 \leq t \in C_{0}^{\infty}(B), t=1$ on $s B$. Then

$$
\begin{aligned}
& \int_{r B}-u \Delta u \leq \int_{B}-t u \Delta u=\int_{B} d t u \wedge d^{c} u \wedge\left(d d^{c}|z|^{2}\right)^{n-1} \\
& \leq\left[\int_{\text {supp } t} d t u \wedge d^{c} t u \wedge\left(d d^{c}|z|^{2}\right)^{n-1}\right]^{1 / 2}\left[\int_{\text {supp } t} d u \wedge d^{c} u \wedge\left(d d^{c}|z|^{2}\right)^{n-1}\right]^{1 / 2}<\infty,
\end{aligned}
$$

which completes the proof of Proposition 1.1.

3. Proof of Lemma 1.2. If $u_{j} \geq u$ then $\widetilde{u}_{j} \geq \widetilde{u}$ so by $(*), \int_{B}-\widetilde{u}_{j} \Delta \widetilde{u}_{j} \leq$ $\int_{B}-\widetilde{u} \Delta \widetilde{u}<\infty$ and

$$
\begin{aligned}
\int_{r B} d\left(u_{j}-u\right) \wedge d^{c}\left(u_{j}-u\right) \wedge & \left(d d^{c}|z|^{2}\right)^{n-1} \\
& \leq \int_{B} d\left(\widetilde{u}_{j}-\widetilde{u}\right) \wedge d^{c}\left(\widetilde{u}_{j}-\widetilde{u}\right) \wedge\left(d d^{c}|z|^{2}\right)^{n-1} \\
& =-\int_{B}\left(\widetilde{u}_{j}-\widetilde{u}\right) d d^{c}\left(\widetilde{u}_{j}-\widetilde{u}\right) \wedge\left(d d^{c}|z|^{2}\right)^{n-1} \\
& \leq \int_{B}\left(\widetilde{u}_{j}-\widetilde{u}\right) d d^{c} \widetilde{u} \wedge\left(d d^{c}|z|^{2}\right)^{n-1} .
\end{aligned}
$$

The last term tends to zero as $j$ tends to infinity and the proof is complete. 
4. The class $\mathcal{E}$. We denote by $\operatorname{PSH}^{-}(\Omega)$ the class of negative plurisubharmonic functions defined on the domain $\Omega$ in $\mathbb{C}^{n}$.

A domain $\Omega$ in $\mathbb{C}^{n}$ is called hyperconvex if there is a negative exhaustion function for $\Omega$, i.e. a function $\psi \in \mathrm{PSH}^{-}(\Omega)$ such that

$$
\{z \in \Omega ; \psi(z)<c\} \subset \subset \Omega, \quad \forall c<0 .
$$

We say that a function $v \in \mathrm{PSH}^{-}(\Omega)$ is in $\mathcal{F}(\Omega)$ if there is a decreasing sequence of functions $v_{j} \in \mathcal{E}_{0}(\Omega)$ with $\lim v_{j}=v$ and $\sup \int\left(d d^{c} v_{j}\right)^{n}<\infty$. Here $\mathcal{E}_{0}(\Omega)$ is the class of bounded plurisubharmonic functions $u$ such that $\lim _{z \rightarrow \xi} u(z)=0$ for all $\xi \in \partial \Omega$ and $\int_{\Omega}\left(d d^{c} u\right)^{n}<\infty$. Finally, $u \in \mathcal{E}(\Omega)$ if for every $\omega \subset \subset \Omega$ there is a function $u \leq u_{\omega} \in \mathcal{F}(\Omega)$ with equality on $\omega$. See $[\mathrm{C} 1, \mathrm{C} 2]$ for further properties of this and related classes.

ThEOREM 4.1. Suppose $\Omega$ is a hyperconvex subset of $\mathbb{C}^{n}$. Then there is a constant $c$, depending on $\Omega$ only, such that if $u \in \mathcal{F}(\Omega)$ then

$$
\int_{\{u<-1\}}|\operatorname{grad} u|^{2} d v \leq c \int_{\Omega}\left(d d^{c} u\right)^{n} .
$$

Proof. By the gradient lemma and Theorem 2.1 in [3], we can assume that $u \in \mathcal{E}_{0}(\Omega) \cap C(\bar{\Omega})$. Then $\{u<-1\} \subset \subset \Omega$. We can choose $m$ and $t$ such that $-1<m\left(|z|^{2}-t\right)<0$ on $\Omega$.

Integration by parts gives

$$
\begin{aligned}
& \int_{\{u<-1\}}|\operatorname{grad} u|^{2} d v=\frac{1}{m^{n-1}} \int_{\{u<-1\}} d u \wedge d^{c} u \wedge\left(d d^{c} m\left(|z|^{2}-t\right)\right)^{n-1} \\
& \leq \frac{1}{m^{n-1}} \int_{\Omega} d u \wedge d^{c} u \wedge\left(d d^{c} \max \left(m\left(|z|^{2}-t\right), u\right)\right)^{n-1} \\
& \quad=\frac{1}{m^{n-1}} \int_{\Omega}-\max \left(m\left(|z|^{2}-t\right), u\right)\left(d d^{c} u\right)^{2} \wedge\left(d d^{c} \max \left(m\left(|z|^{2}-t\right), u\right)\right)^{n-2} \\
& \leq \frac{1}{m^{n-1}} \int_{\Omega} m\left(t-|z|^{2}\right)\left(d d^{c} u\right)^{n} \leq \frac{1}{m^{n-1}} \int_{\Omega}\left(d d^{c} u\right)^{n} .
\end{aligned}
$$

Corollary 4.2. If $u \in \mathcal{E}$, then $u \in \mathrm{PSH}^{-} \cap W_{\mathrm{loc}}^{1,2}$.

Corollary 4.3. Suppose $\Omega$ is a hyperconvex domain in $\mathbb{C}^{2}$. Then there is a constant $c$, depending on $\Omega$ only, such that if $u \in \mathcal{F}(\Omega)$ then

$$
\int_{\Omega}|\operatorname{grad} u|^{2} d v \leq c \int_{\Omega}\left(d d^{c} u\right)^{2} .
$$

TheOREM 4.4 (Błocki [1]). Suppose $\Omega$ is a hyperconvex subset of $\mathbb{C}^{2}$. Then $u \in \mathcal{E}$ if and only if $u \in \mathrm{PSH}^{-} \cap W_{\mathrm{loc}}^{1,2}$. 
Proof. If $u \in \mathcal{E}$, then $u \in \mathrm{PSH}^{-} \cap W_{\text {loc }}^{1,2}$ by Corollary 4.2. Conversely, if $u \in \mathrm{PSH}^{-} \cap W_{\mathrm{loc}}^{1,2}$, then $u \in L_{\mathrm{loc}}^{2}$ and $|\operatorname{grad} u|^{2} \in L_{\mathrm{loc}}^{1}$. Therefore, since $d d^{c} u^{2}=2 d u \wedge d^{c} u+2 u d d^{c} u$, it follows that $d d^{c}\left(u d d^{c} u\right)$ is a well defined positive measure so $u$ is in $\mathcal{E}$.

REMARK. For $u \in \mathcal{F}(\mathbb{B})$, where $\mathbb{B}$ is the unit ball in $\mathbb{C}^{n}, n>1$, we have

$$
\int_{\mathbb{B}}|\operatorname{grad} u|^{2} d v \leq c_{n}^{(n-2) / n}\left[\int_{\mathbb{B}}\left(1-|z|^{2}\right)\left(d d^{c} u\right)^{n}\right]^{2 / n}
$$

where $c_{n}$ is the volume of $\mathbb{B}$.

REMARK. Let $u, w \in \mathcal{F}(\Omega)$ with $\Omega$ hyperconvex. Then, using integration by parts and Theorem 5.5 in [3], we have

$$
\int_{\Omega} d u \wedge d^{c} u \wedge\left(d d^{c} w\right)^{n-1} \leq\left[\int_{\Omega}-w\left(d d^{c} u\right)^{n}\right]^{2 / n}\left[\int_{\Omega}-w\left(d d^{c} w\right)^{n}\right]^{(n-2) / n} .
$$

Choosing $w$ to be a strictly plurisubharmonic function (see e.g. [4]), we get local estimates for $|\operatorname{grad} u|^{2}$.

\section{References}

[1] Z. Błocki, On the general definition of the complex Monge-Ampère operator in $\mathbb{C}^{2}$, Math. Ann. 328 (2004), 415-423.

[2] U. Cegrell, Pluricomplex energy, Acta Math. 180 (1998), 187-217.

[3] - The general definition of the complex Monge-Ampère operator, Ann. Inst. Fourier (Grenoble) 54 (2004), 159-179.

[4] -, Approximation of plurisubharmonic functions in hyperconvex domains, manuscript, Umeå Univ. and Mid Sweden Univ., 2006.

Umeå University

SE-901 87 Umeå, Sweden

E-mail: urban.cegrell@math.umu.se
TFM

Mid Sweden University S-851 70 Sundsvall, Sweden 